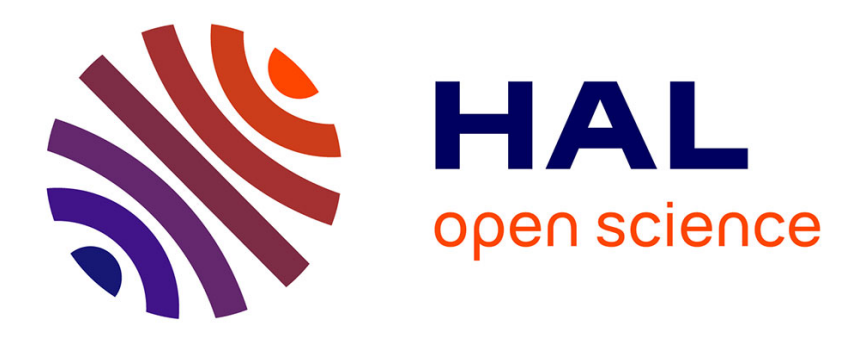

\title{
Nonsimilar Normal Mode Vibrations of Nonlinear Systems Having Two Degrees of Freedom
}

Reinhardt Rosenberg

\section{To cite this version:}

Reinhardt Rosenberg. Nonsimilar Normal Mode Vibrations of Nonlinear Systems Having Two Degrees of Freedom. Journal of Applied Mechanics, 1964, 31 (2), pp.283-290. 10.1115/1.3629599 . hal01344458

\section{HAL Id: hal-01344458 \\ https://hal.science/hal-01344458}

Submitted on 12 Jul 2016

HAL is a multi-disciplinary open access archive for the deposit and dissemination of scientific research documents, whether they are published or not. The documents may come from teaching and research institutions in France or abroad, or from public or private research centers.
L'archive ouverte pluridisciplinaire HAL, est destinée au dépôt et à la diffusion de documents scientifiques de niveau recherche, publiés ou non, émanant des établissements d'enseignement et de recherche français ou étrangers, des laboratoires publics ou privés.

\section{(c)(1)}

Distributed under a Creative Commons Attribution| 4.0 International License 


\section{Nonsimilar Normal Mode Vibrations of Nonlinear Systems Having Two Degrees of Freedom}

When a nonlinear system having several masses vibrates in normal modes, the time histories of the motion of these masses are, in general, different in wave shape (although in certain special nonlinear systems they differ at most in amplitude, but not in shape). When the wave shapes differ, the normal mode vibration is called nonsimilar. In this paper, nonsimilar normal mode vibrations are analyzed with respect to wave shape and stability. The systems considered are those lying close to systems having similar normal mode vibrations. An example is worked out in detail, and a comparison with an experimental study is reported.

IN several earlier papers $[1,2,3,4],{ }^{1}$ the normal mode vibrations of certain nonlinear systems, having many degrees of freedom, have been studied. These systems consist of a chain of masses, each mass having a single degree of freedom of translation in the direction of the chain. Each is connected to others by nonlinear springs, and every spring force is an odd function of the length change of that spring.

The term "normal mode vibration" describes a vibration in unison of all elements of the system, and "vibration in unison" means that all masses execute equiperiodic motions, all pass through equilibrium at the same instant, all attain maximum displacement at the same instant, and the position of any one mass at an $y$ given instant of time defines uniquely that of every other mass at the same instant.

When the system is linear, the motion of every mass during a normal mode vibration is a simple harmonic function of time having the same frequency and the same zeros as every other, or

$$
x_{i}(t) / x_{j}(t)=A_{i} \cos \omega t / A_{j} \cos \omega t \equiv c_{i j}
$$

where $c_{i j}$ is a constant.

When the system is nonlinear, the normal mode vibrations are no longer simple harmonic. Nevertheless, it is quite possible that, even then, the ratios of the displacements in normal mode vibrations are identically equal to constants. In fact, it was shown that symmetric two-degree-of-freedom systems [1] and homogeneous $n$-degree-of-freedom systems $[3,4]$ are such that their normal mode vibrations satisfy

$$
x_{i}(t) / x_{j}(t) \equiv \mathrm{const}
$$

no matter how strongly nonlinear the systems or how large the displacements. The physical interpretation of (1) is that the motions, i.e., the wave shapes in the time-displacement plane, are similar when the system vibrates in normal modes.

While it has also been shown [2] that normal mode vibrations of arbitrary spring-mass systems do not, in general, satisfy (1), all detailed studies have so far been restricted to cases of similar normal mode vibrations.
When the displacements of any two masses $m_{i}$ and $m_{j}$ during normal mode vibrations are such that

$$
x_{i}(t) / x_{j}(t) \not \equiv \text { const }
$$

we shall say that the normal mode vibration is nonsimilar. In this paper, normal mode vibrations of two-degree-of-freedom systems are examined. However, the results can be extended in an obvious way to systems having many degrees of freedom. For this reason, we shall describe a system of many degrees of freedom and specialize it later to the case of two degrees of freedom.

\section{The System}

As a physical model of admissible systems, consider a chain of $n$ mass points $P_{i},(i=1, ., n)$, the mass of $P_{r}$ being $m_{r}$. Since an infinitely large mass can only be accelerated by an infinitely large force, the motion of a mass $m_{q}=\infty$ is $u_{q} \equiv 0$, or $P_{q}$ corresponds to a fixed point. Therefore we may, without increasing the number of degrees of freedom of the system, include a mass $m_{0}=\infty$ in it; such an addition to the system prevents the degree of freedom of "rigid-body translation." Each mass $m_{i},(i=1$, $\ldots, n)$ has a single degree of freedom of translation $u_{i}$ in the direction of the chain. Each is connected to one, several, or all others by springs that may be equal or unequal, linear, nonlinear, or nonlinearizable. ${ }^{2}$ Thus, a system comprising $n$ masses may have as many as $\frac{1}{2}[n(n-1)]$ springs. This system is illustrated in Fig. 1. The corresponding system having two degrees of freedom is shown in Fig. 2.

We assume that there exists at least one configuration in which the system can remain at rest indefinitely. This is the equilibrium configuration and, in the equilibrium configuration, every $u_{i}=0$.

The spring forces are the only forces acting on the system. These are derivable from a potential function $U$ where

$$
U=-V
$$

and $V$ is the potential energy. The potential energy stored in any spring is a function of the absolute value of the length change of that spring. In physical terms, this implies that a spring resists (or aids) in the same degree being deflected by a given amount, whether that deflection is a compression or extension.

If we put $u_{1}=u, u_{2}=v$, the equations of motion of the twodegree-of-freedom system are

$$
m_{1} \ddot{u}=\frac{\partial}{\partial u} U(u, v), \quad m_{2} \ddot{v}=\frac{\partial}{\partial v} U(u, v)
$$

2A spring is said to be "nonlinearizable" if the Taylor expansion of the spring force lacks a linear term. 


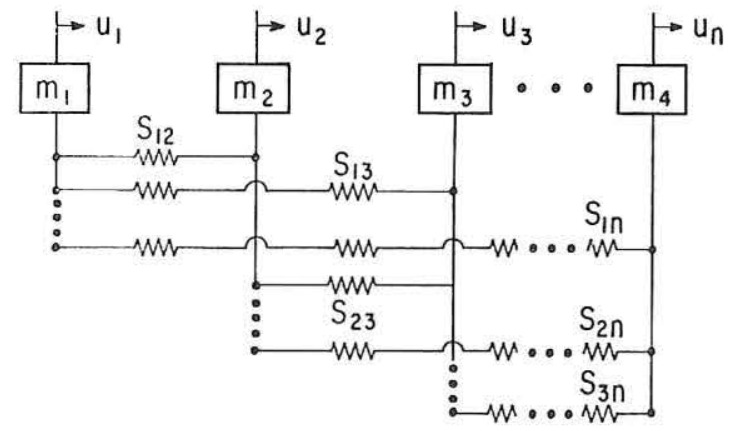

Fig. 1

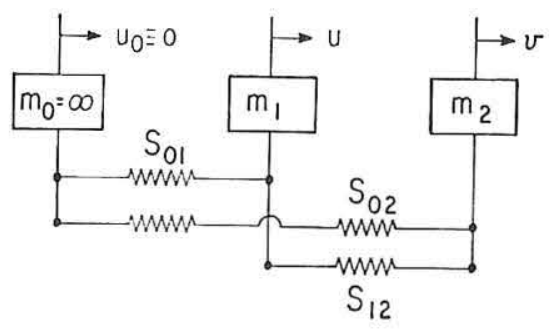

Fig. 2

Substituting

$$
x=m_{2}{ }^{1 / 2} u, \quad y=m_{2}{ }^{1 / 2} v
$$

into (4) one has

$$
\ddot{x}=\frac{\partial}{\partial x} U\left(\frac{x}{m_{2}{ }^{1 / 2}}, \frac{y}{m_{2}^{1 / 3}}\right), \quad \ddot{y}=\frac{\partial}{\partial y} U\left(\frac{x}{m_{1}^{1 / 2}}, \frac{y}{m_{2}^{1 / 2}}\right)
$$

and these may be regarded as the equations of motion of a unit mass that moves in the $x y$-plane under forces $\partial U / \partial x, \partial U / \partial y$. That system of forces and unit mass will be called the pseudosystem. To each motion $u, v$ of the physical system there belongs uniquely a motion of the pseudo-system in the $x y$-plane, and the trajectory of the unit mass satisfies the Euler equations of

$$
\delta \int_{x_{1}}^{x_{2}}(U+h)^{1 / 2}\left(1+y^{\prime 2}\right)^{1 / 2} d x=0
$$

That equation is $[2,5]$

$$
2(U+h) y^{\prime \prime}+\left(1+y^{\prime 2}\right)\left(y^{\prime} U_{x}-U_{y}\right)=0
$$

where primes denote differentiation with respect to $x$.

Inasmuch as the system is conservative, the energy integral

$$
T-U=h
$$

exists where $h$ is a nonnegative constant equal to the total energy and, for the physical and pseudo-systems, respectively,

$$
\left.\begin{array}{ll}
T=\frac{1}{2}\left(m_{1} \dot{u}^{2}+m_{2} \dot{v}^{2}\right) & U=U(u, v) \\
T=\frac{1}{2}\left(\dot{x}^{2}+\dot{y}^{2}\right) & U=U\left(\frac{x}{m_{1}{ }^{1 / 2}}, \frac{y}{m_{2}{ }^{1 / 2}}\right)
\end{array}\right\}
$$

We admit only systems in which $U$ is negative, definite.

\section{Trajectories of Normal Modes}

One sees from (7) that trajectories exist only where $U+h$ is nonnegative, and this is satisfied only in the closed domain $D$ which contains the origin and which is bounded by the closed curve $\Gamma$, defined by

$$
U+h=0
$$

That $\Gamma$ is a closed curve surrounding the origin follows directly from the fact that $U$ is negative definite, and $h$ is nonnegative. The $\Gamma$-curve will be referred to as the bounding curve. It is also the maximum equipotential line because (11) coincides with (9) when $T=0$; i.e., when the velocities vanish, and at such an instant the total energy is potential. Finally, $\Gamma$ is symmetric with respect to the origin [2], and every trajectory which actually intercepts $\Gamma$ does so orthogonally $[2,4,5]$. Solutions of (8) which correspond to vibrations in normal modes are called modal lines.

We shall use perturbation theory to determine the modal lines of systems which lie in the neighborhood of a "parent system" having known modal lines. In this respect, this paper has the same objective as Duffing's memoire [6] had with respect to slightly nonlinear single-degree-of-freedom systems. However, our study is neither restricted to single-degree-of-freedom systems nor to nearly linear ones because the exact modal lines of many highly nonlinear multi-degree-of-freedom systems are known [1, $3,4]$, and the theory to be presented here will hold for the modal lines of any systems in the neighborhood of these nonlinear parent systems.

\section{The Perturbed System}

Let $U=U(x, y)$ be the given potential function of a parent system for which a modal line

$$
y=y^{*}(x)
$$

is known. Further, let

$$
\bar{U}(x, y)=U(x, y)+\epsilon \omega(x, y)
$$

be the potential function of a perturbed system. In (13), $\epsilon$ is a given constant and $\omega(x, y)$ is called the perturbation potential; it is supposedly known. If $|\epsilon|$ is small, and if

$$
\epsilon \omega(x, y)=O(\epsilon)
$$

everywhere in the closed domain $\bar{D}$ bounded by the curve $\bar{\Gamma}$, where the $\vec{\Gamma}$-curve is defined by

$$
\bar{U}+h=U(x, y)+\epsilon \omega(x, y)+h=0
$$

then the perturbed system lies in the neighborhood of the "parent system" whose modal line is (12). We may, then, use first-order perturbation theory to find the modal line

$$
y(x)=y^{*}(x)+\epsilon \eta(x)+\ldots .
$$

everywhere where the equation which (15) must satisfy is regular in $\bar{D}$. In view of (8) and (13), that equation is

$$
y^{\prime \prime}=-\frac{\left(1+y^{\prime 2}\right)\left[y^{\prime}\left(U_{x}+\epsilon \omega_{x}\right)-\left(U_{\nu}+\epsilon \omega_{y}\right)\right]}{2(U+\epsilon \omega+h)}
$$

It is evident that (16) is singular on the $\bar{\Gamma}$-curve and, hence, does not possess a uniformly convergent power series in $\epsilon$ about any point on $\bar{\Gamma}$. Nevertheless, it is always possible to use the perturbation series (15) for constructing solutions that are valid everywhere in the open domain $\bar{D}$, and to continue these solutions analytically until they reach the $\bar{\Gamma}$-curve. The complications arising from the fact that (16) is singular on $\bar{\Gamma}$ will be discussed more fully later on, but first we should like to show that $y^{\prime \prime}$ is hounded ou $\bar{\Gamma}$ even though the denominator of (16) vanishes on it.

Let us suppose that a solution (15) exists which intercepts the $\bar{\Gamma}$-curve at a point $(X, Y)$ where $Y=y(X)$. Then, on $\bar{\Gamma}$,

$$
\dot{x}(X, Y)=\dot{y}(X, Y)=0
$$

because the $\vec{\Gamma}$-curve is defined by (13), and that equation is the energy integral when the kinetic energy vanishes. Then, by l'Hospital's rule,

$$
y^{\prime}(X)=\frac{\dot{y}(X, Y)}{\dot{x}(X, Y)}=\frac{\ddot{y}(X, Y)}{\ddot{x}(X, Y)}=\frac{\bar{U}_{y}(X, Y)}{\bar{U}_{x}(X, Y)}
$$

where use has been made of (6). But (16) is indeterminate when the foregoing value of $y^{\prime}$ is substituted in it; hence, it may also be evaluated by l'Hospital's rule. Making use of 


$$
\begin{aligned}
\frac{d}{d x} \bar{U}_{y}(x, y) & =\bar{U}_{x y}+y^{\prime} \bar{U}_{y y} \\
\frac{d}{d x} \bar{U}_{x}(x, y) & =\bar{U}_{x x}+y^{\prime} \bar{U}_{x y} \\
\frac{d}{d x} \bar{U}(x, y) & =\bar{U}_{x}+y^{\prime} \bar{U}_{y}
\end{aligned}
$$

one finds, after some computations,

$$
y^{\prime \prime}(X, Y)=\frac{1}{3}\left[\frac{\bar{U}_{x y}+y^{\prime}\left(\bar{U}_{y y}-\bar{U}_{x x}-y^{\prime} \bar{U}_{x y}\right)}{\bar{U}_{x}}\right]_{x=X}
$$

By definition of $\bar{U}_{x}(x, y)$, that quantity cannot vanish at $(X, Y)$ [because $(X, Y)$ lies on $\bar{\Gamma}$, and $\bar{\Gamma}$ does not pass through the origin]. Therefore, the denominator of the foregoing equation is not zero. Similarly, the above expression for $y^{\prime}(X)$ shows that that derivative is bounded when $\bar{U}_{x}(X, Y) \neq 0$. It follows that $y^{\prime \prime}(X, Y)$ is bounded, and its value is given in the foregoing equation.

We shall now use first-order perturbation theory to construct a solution of the form (15) which satisfies the equation (16) everywhere inside but not on $\bar{\Gamma}$. Using the notation

$$
Q\left(x, y^{*}(x)\right)=Q^{*}(x)
$$

for $U$ and $\omega$ and for their partial derivatives, standard perturbation technique yields the equation which $\eta(x)$ must satisfy. It turns out to be

$$
\begin{gathered}
2\left(U^{*}+h\right) \eta^{\prime \prime}+\left[2 y^{* \prime}\left(y^{* \prime} U_{x}{ }^{*}-U_{y}{ }^{*}\right)+\left(1+y^{* \prime 2}\right) U_{x}{ }^{*}\right] \eta^{\prime}+ \\
{\left[2 y^{* \prime \prime} U_{y}{ }^{*}+\left(1+y^{* / 2}\right)\left(y^{* \prime} U_{x y}{ }^{*}-U_{y y}{ }^{*}\right)\right] \eta+} \\
2 y^{* \prime \prime} \omega^{*}+\left(1+y^{* \prime 2}\right)\left(y^{* \prime} \omega_{x}{ }^{*}-\omega_{y}{ }^{*}\right)=0
\end{gathered}
$$

But, we seek modal lines lying in the neighborhood of straight ones. Therefore,

$$
y^{*}(x)=c x
$$

For straight modal lines, $y^{* \prime \prime}=0$, and one sees from (7) that, then, everywhere in $\bar{D}$,

$$
y^{* \prime} \equiv U_{y} / U_{x} \equiv c
$$

Substituting (18) and (19) in (17), that equation simplifies considerably and becomes

$$
\begin{aligned}
2\left(U^{*}+h\right) \eta^{\prime \prime}+\left(1+c^{2}\right) U_{x}{ }^{*} \eta^{\prime}+ & \left(1+c^{2}\right)\left(c U_{x y}{ }^{*}-U_{y y}{ }^{*}\right) \eta \\
& =\left(1+c^{2}\right)\left(\omega_{y}{ }^{*}-c \omega_{x}{ }^{*}\right)
\end{aligned}
$$

The integration of this equation is facilitated by the recognition that nearly all coefficients are derivatives of $U^{*}(x)=U\left(x, y^{*}(x)\right)$. For instance,

$$
\begin{aligned}
\frac{d}{d x} U^{*}=U^{*} & =\frac{\partial}{\partial x} U\left(x, y^{*}\right)+\frac{\partial}{\partial y^{*}} U\left(x, y^{*}\right) y^{* \prime} \\
& =U_{x}{ }^{*}+y^{* \prime} U_{y}{ }^{*}
\end{aligned}
$$

and in view of (19),

$$
\frac{d}{d x} U^{*}=\left(1+c^{2}\right) U_{x}^{*}
$$

One finds, similarly, that

$$
\frac{d^{2}}{d x^{2}} U^{*}=\left(1+c^{2}\right) U_{y y}{ }^{*}+\frac{1+c^{2}}{c} U_{x y}{ }^{*}
$$

It follows that, if one defines,

$$
U^{*}(x)+h=G(x)
$$

the perturbation equation (20) becomes

$$
\begin{aligned}
2 G \eta^{\prime \prime}+G^{\prime} \eta^{\prime}-G^{\prime \prime} \eta+\frac{\left(1+c^{2}\right)^{2}}{c} & U_{x y}{ }^{*} \eta \\
& =\left(1+c^{2}\right)\left(\omega_{y}{ }^{*}-c \omega_{x}{ }^{*}\right)
\end{aligned}
$$

It is this equation on which all further development is based.

In certain cases, it may be advantageous to introduce a transformation of the independent variable given by

$$
\xi=\int_{0}^{x} \frac{d u}{G(u)^{1 / 2}}=\psi(x)
$$

This reduces (24) to the form

$2 \frac{d^{2} \eta}{d \xi^{2}}-\left[G^{\prime \prime}-\frac{\left(1+c^{2}\right)^{2}}{c} U_{x y}{ }^{*}\right] \eta=\left(1+c^{2}\right)\left(\omega_{y}{ }^{*}-c \omega_{z}{ }^{*}\right)$

The transformation (25) has an interesting significance. Suppose we wish to determine the time $t$, required for the unit mass of the unperturbed pseudo-system to traverse a given arc of any trajectory in the $x y$-plane. If we denote the velocity of the unit mass by $w$, we find from the energy integral $\frac{1}{2} w^{2}=U+h$ that the transfer time along any trajectory is

$$
\begin{aligned}
t=\int \frac{d s}{w}=\int \frac{d s}{\{2[U(x, y)+h]\}^{1 / 2}} & \\
& =\frac{1}{\sqrt{ } 2} \int \frac{\left(d x^{2}+d y^{2}\right)^{1 / 2}}{[U(x, y)+h]^{1 / 2}}
\end{aligned}
$$

If that trajectory is a straight modal line, so that (18) holds, the transfer time becomes

$$
t=\left(\frac{1+c^{2}}{2}\right)^{1 / 2} \int \frac{d x}{\left(U^{*}+h\right)^{1 / 2}}=\left(\frac{1+c^{2}}{2}\right)^{1 / 2} \int \frac{d x}{G(x)^{1 / 2}}
$$

But this is, except for a constant, the transformation (25). Let us denote by $\tau=\left[2 /\left(1+c^{2}\right)\right]^{1 / 2} t$ the pseudo-time which the unit mass of the unperturbed pseudo-system requires to move from the origin along a straight modal line to a point whose abscissa is $x$. Then the relation between $\xi$ and $x$ is the same as that between $\tau$ and $x$. The times $\tau$ and $t$ coincide when $c= \pm 1$; i.e., for modal lines which are inclined by $45 \mathrm{deg}$ to the $x$-axis.

Equation (26) is simpler in appearance than (24). However, the coefficients in (26) are still functions of $x$, not $\xi$. It is necessary to replace $x$ by $\xi$ in them, before the equation can be integrated; this step is accomplished through the inversion of (25); i.e., by $x=\psi^{-1}(\xi)$.

Equations (24) or (26) are second-order differential equations having solutions of the form

$$
\eta=\eta(x, \alpha, \beta)
$$

The constants of integration $\alpha$ and $\beta$ must be determined such that

$$
y(x)=y^{*}(x)+\epsilon \eta(x, \alpha, \beta)
$$

is a modal line. Since $U(x, y)$ and $\omega(x, y)$ are given functions, and $h, c$, and $\epsilon$ are known constants, the integration of (24) could always be done on a computer.

\section{Integrable Cases}

There exist two cases of considerable physical interest where the perturbation equation can be integrated in terms of quadratures. One of these is the case of "weak coupling" while the second does not require that coupling be weak. We shall discuss these in order.

When the force in the coupling spring is of $O(\epsilon)$ for any finite deflections of that spring, we shall say that the coupling is weak. Such weak coupling has been the subject of many investigations in the theory of nonlinear differential equations [6]. 
The potential energy of the system arises from the potentials $U_{01}, U_{02}, U_{12}$ stored, respectively, in the anchor springs $S_{01}, S_{02}$, and the coupling spring $S_{12}$ (Fig. 2). Clearly, $U_{01}$ is a function of $x$ only, and $U_{02}$ is a function of $y$ only. Hence, their mixed derivatives $U_{x y}$ vanish; in other words, the mixed derivative of the entire system is represented by the mixed derivative $\partial^{2} / \partial x \partial y$ $\left(U_{12}\right)$ of the coupling spring.

When coupling is weak, $U_{x y}$ can only appear in a second-order theory and, hence, will not be present in (24). That equation, then, reduces to

$$
2 G \eta^{\prime \prime}+G^{\prime} \eta^{\prime}-G^{\prime \prime} \eta=\left(1+c^{2}\right)\left(\omega_{y}^{*}-c \omega_{x}^{*}\right)
$$

A first integral of (27) is

$$
\eta^{\prime}=\frac{1}{2 G}\left[G^{\prime} \eta+\alpha-\Omega(x)\right]
$$

where we have used the notation

$$
\Omega(x)=\left(1+c^{2}\right) \int_{0}^{x}\left(c \omega_{x}^{*}-\omega_{y}^{*}\right) d x
$$

and the general solution of (27) is

$$
\eta(x)=\frac{1}{2}[G(x)]^{1 / 2} \int_{0}^{x} \frac{\alpha-\Omega(u)}{G(u)^{3 / 2}} d u+\beta[G(x)]^{1 / 2}
$$

where $\alpha$ and $\beta$ are arbitrary constants of integration.

A second integrable case is that of the in-phase mode of almost symmetric systems, provided the coupling spring is nonlinearizeable or almost nonlinearizable. ${ }^{3}$ However, it is not required that coupling be weak. A system of potential function

$$
U(x, y)=U_{01}(x, y)+U_{02}(x, y)+U_{12}(x, y)
$$

is said to be symmetric if $U_{01}{ }^{*} \equiv U_{02}{ }^{*}$. This requires, in physical terms, that the masses are equal, or $m_{1}=m_{2}$ and that the anchor springs are equal, or $S_{01}=S_{02}$. The system is said to be almost symmetric if $\left|U_{01}{ }^{*}-U_{02}{ }^{*}\right| \equiv O(\epsilon)$. It will now be shown why the in-phase mode of an almost symmetric system is an integrable case when the coupling spring is almost, or altogether, nonlinearizable.

As stated, the mixed derivative $\partial^{2} \bar{U} / \partial x \partial y$ is equal to $\partial^{2} \bar{U}_{12} /$ $\partial x \partial y$. Now, by definition of admissible systems, the potential $\bar{U}_{12}$ is a function of the absolute value $|x-y|$ of the length change. Hence, its Taylor expansion must be of the form

$$
U_{12}=-\sum_{m=1,3, \ldots} \frac{A_{m}}{m+1}(x-y)^{m+1}
$$

and

$$
\frac{\partial^{2} U}{\partial x \partial y}=\sum_{m=1,3, \ldots} m A_{m}(x-y)^{m-1}
$$

But for the in-phase mode of symmetric systems, $y^{*}(x)=x$; hence,

$$
U_{x y}{ }^{*}=A_{1}
$$

Thus, if $A_{1}=0$ or of $O(\epsilon)$, the term $U_{x y}{ }^{*}$ will be absenc from (26). Then, the equation in $\eta$ is (27) with $c=1$, and its general integral is (30), again with $c=1$.

\section{Determination of Constants}

The arbitrary constants $\alpha$ and $\beta$ in (30) define the values of $\eta$ and $\eta^{\prime}$ at the origin. In fact, since $G(0)=h$ in virtue of (23), one has from (30)

$$
\eta(0)=\beta h^{1 / 2}
$$

\footnotetext{
${ }^{3}$ A spring is almost nonlinearizable if the linear component of the spring force is of $O(\epsilon)$.
}

and, since $G^{\prime}(0)=\Omega(0)=0$, the initial slope is, from (28),

$$
\eta^{\prime}(0)=\alpha /(2 h)
$$

These constants must be so determined that $y=y^{*}(x)+\epsilon \eta(x$, $\alpha, \beta)$ is a modal line; i.e., $y(x)$ must pass through the origin, and it must intercept the $\bar{\Gamma}$-curve. From the first condition one has $\beta=0$.

As stated earlier, the perturbation theory used here does not furnish a solution which is valid on $\bar{\Gamma}$; however, the solutions may be continued analytically to $\bar{\Gamma}$.

Let the points whose locus is the $\bar{\Gamma}$-curve be denoted by $(X, Y)$. Then, the equation of the $\bar{\Gamma}$-curve is

$$
U(X, Y)+\epsilon \omega(X, Y)+h=0
$$

The condition that this eurve be intercepted by the analytical continuation of $y(x)$ is satisfied by substituting into (32) the relation

$$
Y=y^{*}(X)+\epsilon \eta(X, \alpha)
$$

where

$$
\eta(X, \alpha)=\frac{1}{2}[G(X)]^{1 / 2} \int_{0}^{X} \frac{\alpha-\Omega(u)}{G(u)^{3 / 2}} d u
$$

This results in an equation of the form

$$
\begin{aligned}
& U\left(X, y^{*}(X)+\epsilon \eta(X, \alpha)\right)+\epsilon \omega\left(X, y^{*}(X)\right. \\
& +\epsilon \eta(X, \alpha))+h=0
\end{aligned}
$$

It is readily seen that this equation will yield a relation of the form

$$
\alpha=\alpha(X)
$$

i.e., an equation connecting the initial slope of $\eta$ with the value $X$ at which the $\bar{\Gamma}$-curve is intercepted, and $X$ is the $x$-amplitude of the motion. In other words, the analytical continuation of every integral curve that lies sufficiently close to the straight modal line of the unperturbed system does intersect the $\bar{\Gamma}$-curve at some point whose abscissa is $X$. Clearly, it is not possible to determine the constant $\alpha$ uniquely from that result. Nevertheless, for future developments, it is necessary to determine the explicit relation between $X$ and $\alpha$.

Let the value of $X$ be given by

$$
X=X_{0}+\epsilon A
$$

where $X_{0}$ is the (known) amplitude of the unperturbed system in the mode whose modal line is $y^{*}=c x$. Let it be assumed further that both the unperturbed and perturbed systems move at the same energy level $h$. Then, the point at which the straight modal line of the unperturbed system intercepts the $\Gamma$-curve is found from

$$
U\left(X_{0}, y^{*}\left(X_{0}\right)\right)+h=0
$$

To find $X$, one must substitute (36) in (34) and expand the resulting equation in powers of $\epsilon$, retaining only terms up to the first power. In doing this, it is necessary to evaluate

$$
\eta\left(X_{0}\right)=\frac{1}{2}\left[G\left(X_{0}\right)\right]^{1 / 2}\left\{\alpha \int_{0}^{X_{0}} \frac{d u}{G(u)^{3 / 2}}-\int_{0}^{X_{0}} \frac{\Omega(u)}{G(u)^{3 / 2}} d u\right\}
$$

Now, $G(x)$ is an even polynomial of at least second degree in $x$ (when the unperturbed problem is linear, otherwise it is of higher degree), and it vanishes at $X_{0}$. The function $\Omega(x)$ is also at least of second degree in $x$; it takes on its maximum value at $X_{0}$ and it vanishes at the origin. Therefore, both integrals in the foregoing equation always diverge; however, their products with $\left[G\left(X_{0}\right)\right]^{1 / 2}$ are bounded, and one can readily find, either from a limit analysis or from l'Hospital's rule,

$$
\eta\left(X_{0}\right)=-\frac{\alpha}{G^{\prime}\left(X_{0}\right)}+\frac{\Omega\left(X_{0}\right)}{G^{\prime}\left(X_{0}\right)}
$$


When (38) is substituted in the expansion of (34) and (37) is introduced in that relation, one finds after some computations

$$
\alpha=\frac{1+c^{2}}{c}\left[A G^{\prime}\left(X_{0}\right)+\omega^{*}\left(X_{0}\right)\right]+\Omega\left(X_{0}\right)
$$

Since $\alpha$ is a measure of the change in initial slope between unperturbed and perturbed problems, and $A$ is a measure of the change in $x$-amplitude between the unperturbed problem and the analytical continuation of the integral curves of the perturbed problem, equations (39) and (36) jointly constitute the explicit form of (35).

We shall now show that there exists at most a single integral curve of the perturbed problem which actually intercepts the $\bar{\Gamma}$-curve; in other words, there exists at most one value of initial slope (i.e., one value of $\alpha$ ) for which an integral curve of the perturbed problem is a modal line.

Let us write, in accordance with (30),

$$
2 \eta=\alpha J(x)-I(x)
$$

where

$$
\begin{gathered}
J(x)=[G(x)]^{1 / 2} \int_{0}^{x} \frac{d u}{G(u)^{3 / 2}} \\
I(x)=[G(x)]^{1 / 2} \int_{0}^{x} \frac{\Omega(u)}{G(u)^{3 / 2}} d u
\end{gathered}
$$

and where we have already put $\beta=0$. Then, the end slope

$$
y^{\prime}(X)=c+\epsilon \eta^{\prime}\left(X_{0}+\epsilon A\right)=c+\epsilon \eta^{\prime}\left(X_{0}\right)
$$

within $O(\epsilon)$, and

$$
2 \eta^{\prime}\left(X_{0}\right)=\lim _{\epsilon_{0} \rightarrow 0}\left[\alpha J^{\prime}\left(X_{0}-\epsilon_{0}\right)-I^{\prime}\left(X_{0}-\epsilon_{0}\right)\right]
$$

Hence, the rate of change of the end slope $\eta^{\prime}\left(X_{0}\right)$ with respect to $\alpha$ is given by

$$
2 \frac{\partial \eta^{\prime}\left(X_{0}\right)}{\partial \alpha}=J^{\prime}\left(X_{0}\right)
$$

and a limit analysis (whose details are not reproduced here) yields

$$
\begin{array}{r}
J^{\prime}\left(X_{0}\right)=\lim _{\epsilon_{0} \rightarrow 0}\left\{\frac { 1 } { \sqrt { \epsilon _ { 0 } } } \left\langle-\frac{1}{2}\left[-G^{\prime}\left(X_{0}\right)\right]^{1 / 2} \int_{0}^{X_{0}-\delta} \frac{d u}{G(u)^{3 / 2}}\right.\right. \\
\left.\left.+1 \sqrt{\delta}\left(-G^{\prime}\left(X_{0}\right)\right)\right\rangle\right\}
\end{array}
$$

where $|\delta|>0$ is a small parameter and $G^{\prime}\left(X_{0}\right)<0$. In consequence, we find

$$
\left|\frac{\partial \eta^{\prime}\left(X_{0}\right)}{\partial \alpha}\right|=\infty
$$

Assume now that an integral exists which is a modal line; i.e., which intercepts the $\bar{\Gamma}$-curve. Then, the last equation states that no other integral curve passing through the origin and having an initial slope neighboring on that of the modal line can also reach the bounding curve because the end slope $\eta^{\prime}\left(X_{0}, \alpha\right)$ is not a continuous function of $\alpha$.

This result is not in contradiction with (39) because that equation holds only for the analytical continuations of the integral curves. Nevertheless, (39) must be satisfied by the actual modal line because that line is the only integral curve which coincides with its analytical continuation.

The condition that $\eta^{\prime}\left(X_{0}\right)$ be bounded, together with the observation that $J^{\prime}\left(X_{0}\right)$ is unbounded might lead one to conjecture that $\alpha=0$; however, that conjecture contains the implicit assumption that $I^{\prime}\left(X_{0}\right)$ is bounded, and such an assumption cannot be verified because $\omega$, and hence $\Omega$, are largely arbitrary.

To determine the initial slope of the modal line of the perturbed system we observe that the left-hand side of (27) becomes self- adjoined when that equation is multiplied by $G(x)^{-1 / 2}$; it then becomes

$2\left(G^{1 / 2} \eta^{\prime}\right)^{\prime}-G^{-1 / 2} G^{\prime \prime} \eta=\left(1+c^{2}\right) G^{-1 / 2}\left(\omega_{y}{ }^{*}-c \omega_{x}^{*}\right) \equiv \varphi(x)(40)$

and the corresponding self-adjoined homogeneous equation is

$$
2\left(G^{1 / 2} \eta^{\prime}\right)^{\prime}-G^{-1 / 2} G^{\prime \prime} \eta=0
$$

One sees from (30), that the general solution of (41) is

$$
\eta=\eta_{0}(x, \alpha, \beta)=\frac{1}{2} G(x)^{1 / 2} \alpha \int_{0}^{x} \frac{d u}{G(u)^{3 / 2}}+\beta G(x)^{1 / 2}
$$

Inasmuch as $\eta$ must pass through the origin, the only case of interest here is that of $\beta=0$, as shown earlier.

Now, it is well known that for existence and uniqueness of solutions of certain inhomogeneous equations, these must satisfy a well-known orthogonality relation [7]; however, because of the unusual "boundary condition" that $y(x)$ intercept a certain curve (rather than that it pass through a given point on a given curve), one must investigate the question of applicability of that orthogonality condition.

This is done by multiplying the inhomogeneous equation (40) by $\eta_{0}(x, \alpha)$ and integrating it over $0 \leqslant x \leqslant X_{0}$, or

$2 \int_{0}^{X_{0}} \eta_{0}\left(G^{1 / 2} \eta^{\prime}\right)^{\prime} d x-\int_{0}^{x_{0}} \eta_{0} G^{-1 / 2} G^{\prime \prime} \eta d x=\int_{0}^{x_{0}} \eta_{0} \varphi d x$

If one integrates the first integral twice by parts, (43) becomes

$$
\begin{aligned}
2\left[G^{1 / 2}\left(\eta_{0} \eta^{\prime}-\eta_{0}{ }^{\prime} \eta\right)\right]_{0}{ }^{X_{0}}+ & \int_{0}^{X_{0}}\left\{\eta \left[2\left(\eta_{0} G^{1 / 2}\right)^{\prime}\right.\right. \\
& \left.\left.-G^{-1 / 2} G^{\prime \prime} \eta_{0}\right]\right\} d x=\int_{0}^{X_{0}} \eta_{0} \varphi d x
\end{aligned}
$$

The first term in equation (44) vanishes in the lower limit because $\eta_{0}(0)=\eta(0)=0$, and in the upper limit because $G^{1 / 2}\left(X_{0}\right)=0$ and we require that the derivatives of $\eta$ and $\eta_{0}$ be bounded at the upper limit.

The integral on the left-hand side of (44) also vanishes because the quantity in the square brackets of the integral is (41), and that quantity is identically zero since $\eta_{0}$ satisfies (41). Hence, the orthogonality condition [7] is applicable; it is

$$
\int_{0}^{X_{0}} \eta_{0} \varphi d x=0
$$

or, explicitly

$$
\int_{0}^{X_{0}} \frac{1}{2} \alpha \int_{0}^{x} \frac{d u}{G(u)^{3 / 2}}\left[\left(1+c^{2}\right)\left(\omega_{y}{ }^{*}-c \omega_{x}^{*}\right)\right] d x=0
$$

Since $G(u) \geqslant 0$ in $0 \leqslant u \leqslant X_{0}$, the integral

$$
\int_{0}^{x} \frac{d u}{G(u)^{3 / 2}}
$$

does not change sign in this interval, and (46) may be written ${ }^{4}$ in the form

$$
\frac{1}{2}\left(1+c^{2}\right)\left(\bar{\omega}_{y}^{*}+c \bar{\omega}_{x}^{*}\right) \alpha \int_{0}^{X_{0}} \int_{0}^{x} \frac{d u}{G(u)^{3 / 2}} d x=0
$$

where

$$
\bar{\omega}_{y}{ }^{*}-c \bar{\omega}_{x}^{*}=\omega_{y}{ }^{*}(\bar{x})-c \omega_{x}{ }^{*}(\bar{x})
$$

and $\bar{x}$ is some value of $x$ in $\left[0, X_{0}\right]$. We cannot impose the constraint

$$
\bar{\omega}_{y}^{*}-c \bar{\omega}_{x}^{*}=0
$$

By the mean-value theory of the integral calculus. 
on $\omega$, because $\omega$ is largely an arbitrary function. For instance when the perturbation consists in perturbing one of the anchor springs only, either $\omega_{y}{ }^{*} \equiv 0, \omega_{x}{ }^{*} \neq 0$, or vice versa; and the nonvanishing quantity never changes sign in $\left[0, X_{0}\right]$. Hence, the orthogonality condition becomes, finally,

$$
\alpha \int_{0}^{X_{0}} \int_{0}^{x} \frac{d u}{G(u)^{3 / 2}} d x=0
$$

But, as siated earlier, $G(x)$ is nonnegative; hence, (48) can only be satisfied by $\alpha=0$.

We, then, have the result that, to the first order in $\epsilon$, the modal line of an integrable, perturbed system is tangent at the origin to that of the unperturbed system on which it neighbors.

In view of this result we have from (30) or (33)

$$
\eta(x)=-\frac{1}{2}[G(x)]^{1 / 3} \int_{0}^{x} \frac{\Omega(u)}{G(u)^{3 / 2}} d u
$$

and, from (39), the change in $x$-amplitude between unperturbed and perturbed systems is defined through

$$
A=-\frac{1}{G^{\prime}\left(X_{0}\right)}\left[\omega^{*}\left(X_{0}\right)+\frac{c}{1+c^{2}} \Omega\left(X_{0}\right)\right]
$$

The solutions of unperturbed and perturbed systems are illustrated in Fig. 3. From this diagram, one sees that the perturbation has first-order effects on the $x$ and $y$-amplitudes of the normal mode vibrations.

A measure of the change in $y$-amplitude is found from

$$
\begin{aligned}
y(X)=Y & =y^{*}(X)+\epsilon \eta(X) \\
= & y^{*}\left(X_{0}+\epsilon A\right)+\epsilon \eta\left(X_{0}+\epsilon A\right)
\end{aligned}
$$

Expanding the last equation in porvers of $\epsilon$ up to the first, and making use of $y^{* \prime}(x)=c$, one has

$$
Y=Y_{0}+\epsilon\left[c A+\eta\left(X_{0}\right)\right]
$$

where $Y_{0}=y^{i}\left(X_{0}\right)$ is the $y$-amplitude of the unperturbed system. If we write, similar to (36),

$$
Y=Y_{0}+\epsilon B
$$

we have

$$
B=c A+\eta\left(X_{0}\right)
$$

where $A$ is given in (50), and $\eta\left(X_{0}\right)$ in (38) (with $\alpha=0$ ). Making use of these, one finds

$$
B=\stackrel{\text { " }}{-} \frac{1}{G^{\prime}\left(X_{0}\right)}\left[c \omega^{*}\left(X_{0}\right)-\frac{1}{1+c^{2}} \Omega\left(X_{0}\right)\right]
$$

The result that the perturbation has first-order effects on the amplitudes is at odds with those of Huang [8] who examined the nearly linear dynamic vibration absorber with a slightly nonlinear coupling spring. In our terminology, Huang's problem lies in the neighborhood of a homogeneous system of degree 1. Huang

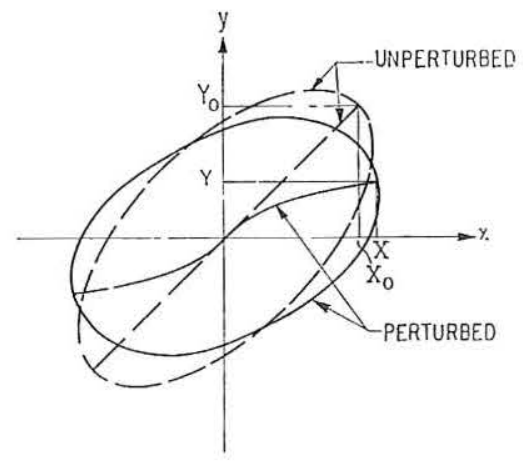

Fig. 3 used standard perturbation techniques on the equations of motion, not on the geometrical equation (8) used here, and concluded that the vibration amplitudes of the perturbed system are in the same ratio to each other as those of the unperturbed problem. That Huang's results are incorrect was shown recently by Szemplinska-Stupnicka [9] who showed that, in fact, certain first-order effects are neglected in Huang's analysis, and that these can only be recovered by a second-order analysis (that one in turn losing certain second-order effects which must be recovered through a third-order analysis, and so forth). However, when using the geometrical methods applied here, all first-order effects are accounted for by a first-order analysis.

\section{The Motion and Its Stability}

Once the modal line $y(x)$ of the perturbed system has been found by the methods described here, the motion of the perturbed system in the normal modes can be found by a simple quadrature. The substitution of the modal line

$$
y=y^{*}(x)-\frac{1}{2} \epsilon[G(x)]^{1 / 2} \int_{0}^{x} \frac{\Omega(u)}{G(u)^{3 / 2}} d u
$$

into the first of (6) gives

$$
\ddot{x}=\bar{U}_{x}(x, y(x))=U_{x}(x, y(x))+\epsilon \omega_{x}(x, y(x))
$$

Expansion within $\mathrm{O}(\epsilon)$ of (54) results in

$$
\ddot{x}=U_{x}{ }^{*}(x)+\epsilon\left[\eta(x) U_{x y}{ }^{*}+\omega_{x}^{*}(x)\right]
$$

But, in the integrable cases, $U_{x y}{ }^{*}$ is either of $O(\epsilon)$ or zero, so that the foregoing equation becomes

$$
\ddot{x}=U_{x}^{*}(x)+\epsilon \omega_{x}^{*}(x)
$$

This equation must be solved for the initial conditions

$$
x(0)=X_{0}+\epsilon A, \quad \dot{x}(0)=0
$$

where $A$ is given in (50). The solution may be found by one quadrature and is of the form

$$
x=\varphi(t, A)
$$

It is expressly noted that (55), and hence (56), is free of $\eta$. The $y$-motion is

$$
\begin{aligned}
y & =y^{*}(\varphi(t, A))+\epsilon \eta(\varphi(t, A)) \\
& =c \varphi(t, A)+\epsilon \eta(\varphi(t, A))
\end{aligned}
$$

and (57) does contain $\eta$. Since (57) depends on $\eta$ and (56) does not, the normal mode vibration is, in general, nonsimilar.

Next we show that the stability of the normal mode vibration of the perturbed system is the same as that of the unperturbed system.

The solutions whose stability is to be examined are

$$
\begin{aligned}
& x=\varphi(t) \\
& y=c \varphi(t)+\epsilon \eta(\varphi(t)) \equiv \psi(t)
\end{aligned}
$$

and the equations, identically satisfied by (58), are

$$
\begin{aligned}
& \ddot{x}=U_{x}(x, y)+\epsilon \omega_{x}(x, y) \\
& \ddot{y}=U_{y}(x, y)+\epsilon \omega_{y}(x, y)
\end{aligned}
$$

Let the solutions of the perturbed system (59), but with slightly different initial values than (58), be

$$
\begin{aligned}
& x=\varphi(t)+\epsilon_{0} p(t) \\
& y=\psi(t)+\epsilon_{0} q(t)
\end{aligned}
$$

Then the solutions (58) are said to be stable if $p(t)$ and $q(t)$ are bounded for all $t$.

The equations of the first variation of (59) with respect to (58) are readily computed; they are 


$$
\begin{aligned}
& p=p U_{x x}(\varphi, \psi)+q U_{z y}(\varphi, \psi) \\
& \ddot{q}=p U_{x y}(\varphi, \psi)+q U_{y y}(\varphi, \psi)
\end{aligned}
$$

But these are precisely the variational equations of the unperturbed system. Hence, the stability of the normal mode solutions of the two systems is the same. The stability problem is now solved because the stability of the unperturbed problem is deducible by known methods $[1,3,10,11]$.

\section{Example}

An an example of the foregoing theory, we shall consider an almost symmetric system consisting of two masses and three springs, as shown in Fig. 2. The symmetry is disturbed by a slight change in the spring $S_{02}$ which connects the mass $m_{2}$ to the fixed point. This change is to be such that the force of the perturbed spring remains an odd function of the spring deflection. Consequently,

$$
\begin{aligned}
\omega(x, y) & =f(y) \\
f(-y) & =-f(y) \\
f(0) & =0
\end{aligned}
$$

The change in the bounding curve resulting from this perturbation is readily discussed. Because of the third of (62), the perturbed bounding curve is tangent to that of the unperturbed problem where $y=0$, i.e., where both cross the $x$-axis. If the perturbation strengthens the spring $S_{02}$, the $\bar{\Gamma}$-curve lies inside the $\Gamma$-curve for every $y \neq 0$; if the perturbation weakens $S_{42}, \bar{\Gamma}$ lies outside $\Gamma$ for every $y \neq 0$.

We shall examine the in-phase mode of the perturbed system. Then, since the parent system is symmetric, $y^{*}=x$ or $c=1$ [1], and

$$
\begin{array}{ll}
\omega^{*}(x)=f(x), & \omega_{y}=f^{\prime}(y) \\
\omega^{*}\left(X_{0}\right)=f\left(X_{0}\right), & \omega_{y}{ }^{*}=f^{\prime}(x) \\
\omega_{x} \equiv 0, & \Omega\left(X_{0}\right)=-2 \int_{0}^{X_{0}} f^{\prime}(x) d x=-2 f\left(X_{0}\right)
\end{array}
$$

Substituting these quantities in (50), one has (because of $c=1$ ) $A=0$. Therefore, under the proposed perturbation, the $X$-amplitude remains unchanged.

The change in $y$-amplitude is found by substituting the foregoing quantities in (52). The result is

$$
B=-\frac{2 f\left(X_{0}\right)}{G^{\prime}\left(X_{0}\right)}
$$

Now, $G^{\prime}\left(X_{0}\right)<0$ for all $0<x \leqslant X_{0}$. Hence, $B$ has the same sign as $f\left(X_{0}\right)$. If the perturbation is such as to strengthen the spring $S_{02}, f(x)<0$ for all $0<x \leqslant X_{0}$; therefore, the $y$-amplitude will be diminished. If the perturbation weakens $S_{02}, f(x)>0$, and the $y$-amplitude will be increased. Both cases are illustrated in Fig. 4.

If it is the spring $S_{01}$ which is perturbed rather than $S_{02}$, one has $\omega_{x}=\omega_{y}{ }^{*}=f^{\prime}(x)$ and $\omega_{y} \equiv 0$. Then, in view of $(29), \Omega\left(X_{0}\right)=$ $2 f\left(X_{0}\right)$, and one finds as expected

$$
A=-\frac{2 f\left(X_{0}\right)}{G^{\prime}\left(X_{0}\right)}, \quad B=0
$$

In that case, the $\bar{\Gamma}$ and $\Gamma$-curves are tangent to each other on the $y$-axis, and $\bar{\Gamma}$ lies inside $\Gamma$ for $f(x) \leqslant 0$, and outside for $f(x) \geqslant 0$.

The first of these results has also been checked experimentally on the analog computer. The unperturbed system chosen in the experiment was a symmetric, homogeneous system of degree 3 and with potential function

$$
U(x, y)=-\frac{1}{2}\left(x^{4}+y^{4}\right)-\frac{1}{2}(x-y)^{4}
$$

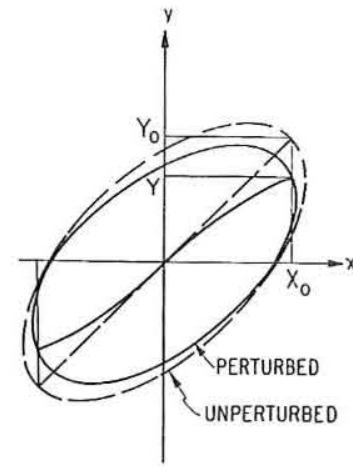

SPRING $\mathrm{S}_{02}$ STRENGTHENED

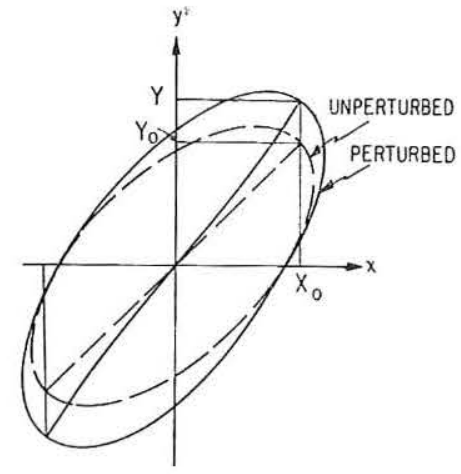

SPRING $\mathrm{S}_{02}$ WEAKENED

Fig. 4

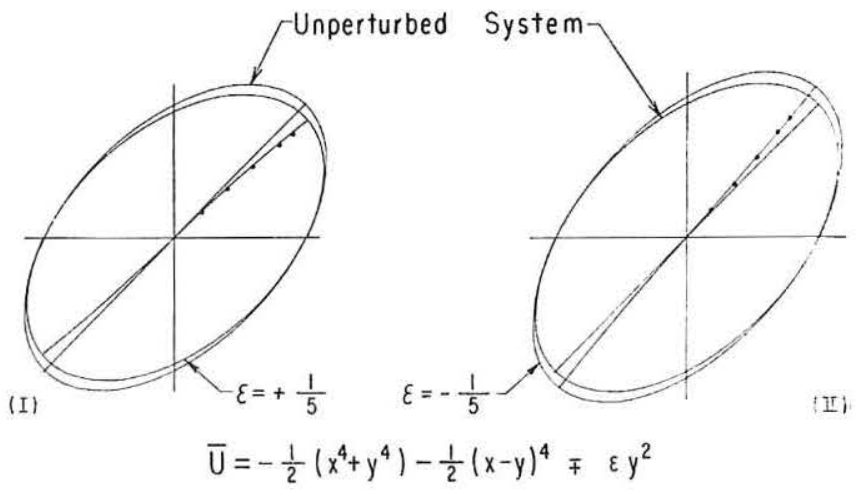

- Drawn by computer for actual nonlinear system

Numerical results of first order perturbotion theory

Fig. 5

The perturbation of the spring $S_{02}$ consisted of the addition of a small linear term which perturbed the homogeneity as well as the symmetry. In the case of a strengthened spring, the perturbation potential was

$$
\epsilon \omega(x, y)=-\frac{1}{5} y^{2}
$$

and, in the case of a weakened $S_{02}$, the negative of the foregoing function was used. The resulting curves in the $x y$-plane are shown in Fig. 5 for both the unperturbed and the perturbed systems. Thus, these curves are the experimental counterparts of Fig. 4. All lines in Fig. 5, including the axis system, were recorded by an $x y$-plotter and only the lettering was added by hand. A comparison between Figs. 4 and 5 shows that the agreement is excellent even though the perturbation was much larger than contemplated in a first-order theory. Quantitatively, the difference between the predicted and observed amplitude changes is hardly measurable.

\section{Acknowledgment}

Financial aid from the National Science Foundation which has supported this work is gratefully acknowledged.

\section{References}

1 R. M. Rosenberg and C. P. Atkinson, "On the Natural Modes and Their Stability in Nonlinear Two-Degree-of-Freedom Systems," Journal of Applied Mechanics, vol. 26, Trans. ASME, vol. 81, Series E, 1959, pp. 377-385.

2 R. M. Rosenberg, "Normal Modes of Nonlinear Dual-Mode Systems," Journal of Applied Mechanics, vol. 27, Trans. ASME, vol. 81, Series E, 1960, pp. 263-268.

3 R. M. Rosenberg, "On Normal Vibrations of a General Class of Nonlinear Dual-Mode Systems," Journal of Applied ME- 
chanics, vol. 28, Trans. ASME, vol. 82, Series E, 1961, pp. 275283.

4 R. M. Rosenberg, "The Normal Modes of Nonlinear n-Degreeof-Freedom Systems," Journal of Applied Mechanics, vol. 29 Trans. ASME, vol. 83, Series E, 1962, pp. 7-14.

5 H. Kauderer, Nichtlineare Mechanik, Springer-Verlag, Berlin, Göttingen, Heidelberg, 1958, p. 599

6 Jules Haag, Oscillatory Motions, English translation, Wadsworth Publishing Company, Inc., Englewood Cliffs, N. J., vol. 2, 1962 , pp. 66 and 157 .

7 R. Courant and D. Hilbert, Methoden der Mathematischen Physik, Springer-Verlag, Berlin, Germany, vol. 1, 1931, p. 307.

8 T. C. Huang, "Harmonic Oscillations of Nonlinear Two-
Degree-of Freedom Systems," Journal of Applied Mechanics, vol. 22, Trans. ASME, vol. 77, Series E, 1955, pp. 107-110.

9 Wanda Szemplinska-Stupnicka, "Normal Modes of a Nonlinear Two-Degree-of-Freedom System and Their Properties," Proceedings, Second International Conference on Nonlinear Vibrations,

Warsaw, Poland, 1962 (to appear).

10 R. M. Rosenberg and C. S. Hsu, "On the Geometrization of Normal Vibrations of Nonlinear Systems Having Many Degrees of Freedom," Proceedings, IUTAM Symposium on Nonlinear Vibrations, Kiev, 1961 (to appear).

11 C. S. Hsu, "On a Restricted Class of Coupled Hill's Equations and Some Applications," Journal of Applied Mechanics, vol. 28 Trans. ASME, vol. 82, Series E, 1961, pp. 551-556. 